\title{
Metabolic Engineering of Monoterpene Synthesis in Yeast
}

\author{
Marc J. C. Fischer, ${ }^{1}$ Sophie Meyer, ${ }^{1}$ Patricia Claudel, ${ }^{1}$ Marc Bergdoll, ${ }^{2}$ Francis Karst $^{1}$ \\ ${ }^{1}$ Univ Strasbourg, INRA, Inst Natl Recherche Agron, Métab Second Vigne, Unit Mixte \\ Recherche Santé Vigne \& Qual Vins, 28 rue de Herrlisheim, F-68021Colmar, France; \\ telephone: 33-3-89-22-49-81; fax: 33-3-89-22-49-89; e-mail: fischer@colmar.inra.fr \\ ${ }^{2}$ The Inst Biol Mol Plantes, Univ Strasbourg, CNRS, F-67084 Strasbourg Cedex, France \\ Received 17 November 2010; revision received 8 February 2011; accepted 3 March 2011 \\ Published online 9 March 2011 in Wiley Online Library (wileyonlinelibrary.com). DOI 10.1002/bit.23129
}

\begin{abstract}
Terpenoids are one of the largest and most diverse families of natural compounds. They are heavily used in industry, and the trend is toward engineering modified microorganisms that produce high levels of specific terpenoids. Most studies have focused on creating specific heterologous pathways for sesquiterpenes in Escherichia coli or yeast. We subjected the Saccharomyces cerevisiae ERG20 gene (encoding farnesyl diphosphate synthase) to a set of amino acid mutations in the catalytic site at position K197. Mutated strains have been shown to exhibit various growth rate, sterol amount, and monoterpenol-producing capacities. These results are discussed in the context of the potential use of these mutated strains for heterologous expression of monoterpenoid synthases, which was investigated using Ocimum basilicum geraniol synthase. The results obtained with up to $5 \mathrm{mg} / \mathrm{L}$ geraniol suggest a major improvement compared with previous available expression systems like Escherichia coli or yeast strains with an unmodified ERG20 gene that respectively delivered amounts in the 10 and $500 \mu \mathrm{g} / \mathrm{L}$ range or even a previously characterized K197E mutation that delivered amounts in the $1 \mathrm{mg} / \mathrm{L}$ range.

Biotechnol. Bioeng. 2011;108: 1883-1892.

(C) 2011 Wiley Periodicals, Inc.

KEYWORDS: terpenes; geraniol; erg20; FPPS; Saccharomyces cerevisiae
\end{abstract}

\section{Introduction}

Isoprenoids comprise a very large family with over 40,000 molecules identified till date (Withers and Keasling, 2007). They are classified by the number of carbons in their skeletal structure: hemi (C5), mono- (C10), sesqui- (C15), di(C20), and tri- (C30) terpenoids. Some terpenoids are of economic importance as bioactive compounds in medicine,

Correspondence to: Marc J. C. Fischer flavors or fragrances for the food and fragrance industry, with market values of around $\$ 1$ billion (Kirby and Keasling, 2009). Some of these compounds are naturally encountered in plants at low concentrations and are considered attractive targets for bioengineering. Much work has focused on the heterologous production of sesquiterpenes in Escherichia coli and Saccharomyces cerevisiae. However, such production is not straightforward, and augmenting the usually disappointing levels now tends to be done via engineering the isoprenoid pathway, or heterologous expression of several enzymes to reconstitute a partial isoprenoid pathway (Martin et al., 2003; Shiba et al., 2007; Takahashi et al., 2007). Monoterpene synthases have been studied less than sesquiterpene synthases and very little work has been done to optimize E. coli or S. cerevisiae strains to generate a pool of available geranyl diphosphate (GPP) that would enable efficient monoterpene production (Oswald et al., 2007; Reiling et al., 2004).

With the exception of a few winemaking strains able to produce up to $5 \mu \mathrm{g} / \mathrm{L}$ monoterpenes, wild $S$. cerevisiae reportedly does not make monoterpenes (Carrau et al., 2005; Zea et al., 1995). Unlike plants, microorganisms usually do not carry a specific GPP synthase (GPPS). In yeast, both GPP and farnesyl diphosphate (FPP) synthase activities are shared by one enzyme called farnesyl diphosphate synthase (FPPS). Consequently, they cannot be easily separated. It was thought that GPP was not released from the catalytic site, and was entirely converted to FPP. FPP is the precursor of several classes of essential yeast metabolites which are utilized for several biosynthetic pathways such as those of ergosterol, dolichols, ubiquinone, heme A, prenylated proteins (Grabinska and Palamarczyk, 2002; Grabinska et al., 2007; Szkopinska and Plochocka, 2005), whereas no specific role has been assigned to GPP besides being a precursor of FPP. The FPPS gene is essential for S. cerevisiae as gene disruption is lethal (Anderson et al., 1989). The yeast FPPS gene encodes a $40.5-\mathrm{kDa}$ polypeptide of 342 amino acids with a high degree of similarity to FPPS 
from other organisms, as well as to various enzymes of the trans-prenyltransferase protein family, such as GPPS and geranylgeranyl diphosphate synthase (GGPPS) (Ye et al., 2007). The yeast FPPS catalyses the formation of the C15 FPP by two sequential condensation reactions of isopentenyl diphosphate (IPP) with dimethylallyl diphosphate (DMAPP) into GPP, and then GPP with another IPP molecule into FPP. The necessity of FPPS for yeast survival and the enzyme's ability to produce only FPP left little room for technological improvement. However it was possible to isolate a yeast mutant exhibiting a 14-fold lower FPPS-specific activity (Chambon et al., 1990). The mutated gene $\operatorname{erg} 20-2$ showed a single nucleotide change, resulting in a K[197] $\rightarrow$ E substitution in FPPS (Blanchard and Karst, 1993). It is worth noting that this K197 is a highly conserved residue amongst FPPS of various origins; a PipeAlign multialignment (Plewniak et al., 2003) made with about 200 available sequences showed no divergent possibility. This mutant was studied for physiological properties and shown to have several unusual properties such as excreting prenyl alcohols geraniol and linalool (Chambon et al., 1990), and synthesizing significantly longer dolichols than the wild type strain FL100 (14-31 and 14-19 isoprene units, respectively) (Plochocka et al., 2000). An additional set of mutations (K197R, K197E and K197V) showed a correlation between the FPPS activity, the amount of ergosterol synthesized and cell growth (Karst et al., 2004).

The presence of geraniol in this strain prompted us to suspect GPP release from the FPPS and a conversion of GPP to geraniol. We first had to make assumptions as to how this release could occur. The crystal structure of avian FPPS presented by Tarshis et al. (Berman et al., 2000; Tarshis et al., 1994) enabled us to construct a model of yeast FPPS. Sequence alignment of FPPS and GGPPS from many different organisms revealed several conserved regions with two characteristic Asp-rich motifs. The first region has a DDXXD or DDXXXXD motif called FARM (first Asp-rich motif), which has been designated as the chain length determination (CLD) region and binds the allylic substrates DMAPP, GPP or FPP, depending on the reaction catalyzed (Koyama et al., 1993; Stanley Fernandez et al., 2000). The second motif has the DDXXD sequence called SARM (second Asp-rich motif) and binds homoallylic isopentenyl diphosphate (Ashby and Edwards, 1990; Szkopinska and Plochocka, 2005). The two binding sites are spatially close. Farnesyl diphosphate synthase works as a dimer. The crystal structure of avian FPPS has shown that both subunits are related by a perfect two-fold axis and in each of them, 10 core helices are arranged around a large central cavity. (Tarshis et al., 1994). This study was later completed by the determination of several ligand-bound and unliganded FPPS structures (Vandermoten et al., 2009). The catalytic site of the enzyme consists of the large central cavity formed by mostly antiparallel R-helices with two aspartate-rich FARM and SARM regions located on opposite walls. The aspartates mediate the condensation reactions of IPP with the allylic substrates by bridging three $\mathrm{Mg}^{2+}$ ions to the protein (Tarshis et al., 1996). The mutated FPPS we isolated did not contain a mutation in these catalytic domains previously described. Our model showed that yeast K197, the amino acid equivalent to avian $\mathrm{K} 214$, is in the active center of the enzyme between two aspartate-rich motifs (Plochocka et al., 2000) and is involved in $\mathrm{Mg}^{2+}$ coordination (Fig. 1). Glutamic acid lacks the $\varepsilon$-amino group of lysine which, as suggested for avian K214, may function in

\section{A}

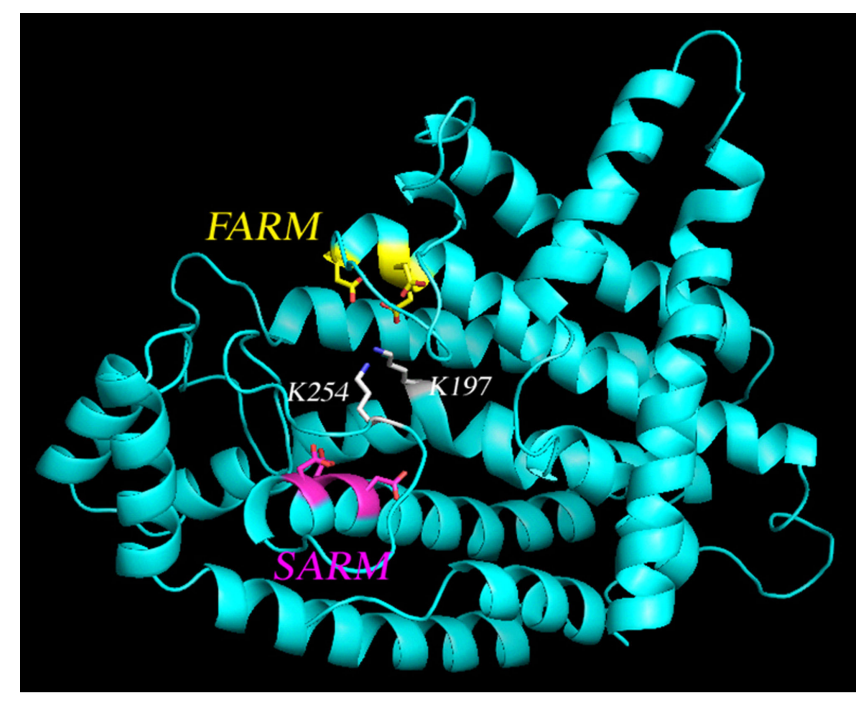

B

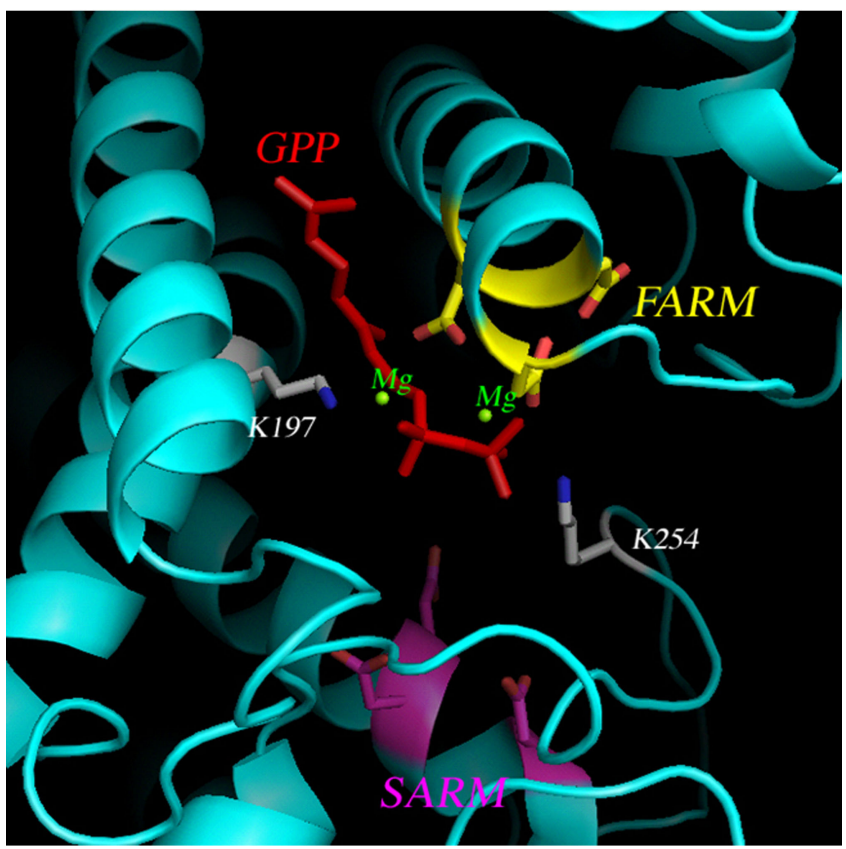

Figure 1. FPPS catalytic site. Positions K197. K 254. FARM and SARM domains in the catalytic site are shown in color (A). Spatial interaction of GPP and $\mathrm{Mg}^{2+}$ with $\mathrm{K} 197$ and $\mathrm{K} 254$ in the catalytic site is detailed (B). 
catalysis by activation of the diphosphate leaving group, and perhaps in subsequent stabilization of the carbocation intermediates and deprotonation of C2 of IPP (Bitsch et al., 2006; Hosfield et al., 2004; Sanchez et al., 2006; Tarshis et al., 1994). In our model, E increased the depth of the substrate-binding pocket and consequently, the activity of the K197E yeast's mutated FPPS is lowered (Plochocka et al., 2000).

At that stage we knew that the tight binding of GPP to the farnesyl diphosphate synthase (FPPS) catalytic site can be disturbed under some circumstances, as it was possible to isolate mutants excreting geraniol as well as increase the amount up to about $1 \mathrm{mg} / \mathrm{L}$ by the heterelogous expression of a geraniol synthase (GES) from basilic (Oswald et al., 2007). These results prompted us to introduce a complete set of defined mutations at position K197 into the ERG20 gene to obtain a set of yeast mutant strains potentially differing in GPPS and FPPS activities and to assess whether their monoterpenol production would differ upon expression of an heterologous monoterpene synthase. As yeast is one of the best-known heterologous models for expressing animal or plant genes, we decided to make a closer assessment of our strains' potential for monoterpenol biosynthesis by evaluating the phenotypic effects of both mutations and monoterpenol synthase expression on cell viability and gross metabolism.

\section{Materials and Methods}

\section{Yeast Strains Construction}

Yeast strains were grown aerobically in complete YPD medium or in minimal medium (1.7 g/L Yeast Nitrogen Base (Difco), $5 \mathrm{~g} / \mathrm{L}$ ammonium sulfate (Merck) supplemented with $1 \%$ glucose as carbon source and required amino acids (histidine, leucine, uracil) at $50 \mu \mathrm{g} / \mathrm{mL}$ or G418 at $200 \mu \mathrm{g} / \mathrm{mL}$ (Euromedex)). The Euroscarf Y21258 diploid strain (Mat a/ $\alpha$, his $3 \Delta 1 /$ his $3 \Delta 1$, leu $2 \Delta 0 /$ leu $2 \Delta 0$, lys $2 \Delta 0 / L Y S 2$, MET15/met15 $\Delta 0$, ura 3 $\Delta 0 /$ ura3 $\Delta 0$; YJL167W::kanMX4/ YJL167W) bearing one deleted allele of ERG20 was transformed by electroporation with plasmid pFL44ERG20 to generate AE101 diploid strain (Becker and Guarante, 1991).

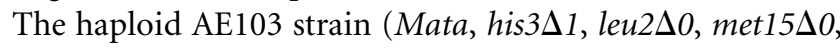

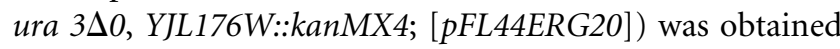
from AE101 sporulation on SP1 medium (1\% potassium acetate (Prolabo), 0.1\% Yeast Extract (Difco), 0.05\% glucose (Euromedex), 2\% agar (Euromedex)) supplemented with leucine (Euromedex) and histidine (Euromedex)) and microdissection. AE103 strain was crossed with Fy $\alpha$ (Mat $\alpha$; his3 $\Delta 200$, leu $2 \Delta 1, \operatorname{trp} 1 \Delta 63$, ura3-52) to introduce Trp1 auxotrophy. The haploid AE9 strain (Mat $\alpha$, his3 $\Delta$, leu $2 \Delta 0$, ura3-, trp1s63, YJL167W::kanMX4 [pFL44ERG20]) was selected after sporulation on SP1 medium supplemented with histidine, leucine, tryptophane (Euromedex) and microdissection of the haploid spores.

\section{Site Directed Mutagenesis of FPPS}

Replicative plasmid pLB41, carrying FPPS ORF under the control of its own promoter and terminator sequences, was used for site directed mutagenesis of yeast FPPS (Blanchard and Karst, 1993). The plasmids carrying the K197 FPPS mutations were created using the QuikChange SiteDirected Mutagenesis Kit from Stratagene following the manufacturer's instructions. The Forward and Reverse complementary primers were designed: CATAGTTACT TTC123ACTGCTTACTATTCTTTCTACTTGCC. The positions labeled 123 correspond to the AAG codon mutated to generate the set of K197 mutations (G(GGT) - A(GCG) V(GTG $)$ - L(CTA $)$ - I(ATC) - P(CCG $)$ - F(TTC) - Y (TAC) - W(TGG) - C(TGC) - M(ATG) - S(TCG) - T(ACT) $\mathrm{K}(\mathrm{ATC})-\mathrm{R}(\mathrm{CGG})-\mathrm{H}(\mathrm{CAC})-\mathrm{D}(\mathrm{GAT})-\mathrm{E}(\mathrm{GAG})-$ $\mathrm{N}(\mathrm{AAC})$ - $\mathrm{Q}(\mathrm{CAA}))$. All plasmids were verified by sequencing.

\section{Yeast Transformation and Plasmid Shuffle}

Characterization of the mutated genes was done by expression in yeast devoid of chromosomal ERG20 gene, constructed by plasmid shuffling. A $1.5 \mathrm{~mL}$ aliquot of AE9 cells grown to stationary phase in YPD medium was centrifuged ( $1 \mathrm{~min}, 10,000 \mathrm{~g}$ ) and $125 \mathrm{ng}$ of mutated plasmid pLB41 was added with $50 \mu \mathrm{g}$ single stranded salmon sperm DNA as carrier to the yeast pellet. Transformed cells were selected for trytophane prototrophy and G418 resistance on minimal medium. pFL44ERG20 plasmid loss was induced at $28^{\circ} \mathrm{C}$ for 3-7 days depending on strain growth onto a minimal medium plate containing $700 \mu \mathrm{g} / \mathrm{mL}$ 5-fluoroorotic acid (ABI), $80 \mu \mathrm{g} / \mathrm{mL}$ ergosterol (Sigma) and the adequate amino acids. The strains thus generated correspond to the first set of strains used for this study. The FPPS mutated strains were then transformed with pMO5 plasmid expressing Ocimum basilicum GES in fusion with green fluorescent protein (GFP) (Oswald et al., 2007).

\section{Yeast Growth Assays}

A $24 \mathrm{~h}$ preculture $(10 \mu \mathrm{L})$ was used to inoculate a $400 \mu \mathrm{L}$ filter-sterilized YNB medium supplemented with the adequate auxotrophic amino acids. Yeast cells were grown for 7-10 days in a Bioscreen C (Oy Growth Curves Ab Ltd., Helsinki, Finland) at $28^{\circ} \mathrm{C}$ and with continuous shaking. Absorbance at $600 \mathrm{~nm}$ was measured every hour.

\section{Sterol Extraction and Quantification}

Yeast cells were grown in a shaking incubator at $30{ }^{\circ} \mathrm{C}$ in 500-mL Erlenmeyer flasks containing $150 \mathrm{~mL}$ of liquid minimal medium. Auxotrophic requirements were supplied as required at $50 \mu \mathrm{g} / \mathrm{mL}$. Sterol extraction after saponification of lyophilized cells and analysis were performed as previously described (Marcireau et al., 1990). $\Delta-5,7$ sterols 
(ergosterol and ergosta-5,7-dienol) were quantified by measuring the UV absorbance at $281.5 \mathrm{~nm}$ (Servouse and Karst, 1986). Sterol composition was determined by GC (Trace GC, Thermo Electron equipped with a on-column injector and a flame ionization detector) under the following conditions (capillary column SPB5 Supelco $15 \mathrm{~m}, 0.32-\mathrm{mm}$ ID, $0.25-\mathrm{mm}$ film thickness, carrier gas helium, $1.3 \mathrm{~mL} /$ min): $60{ }^{\circ} \mathrm{C}$ for $1 \mathrm{~min}, 30^{\circ} \mathrm{C} / \mathrm{min}$ to $220^{\circ} \mathrm{C}, 3{ }^{\circ} \mathrm{C} / \mathrm{min}$ to $290^{\circ} \mathrm{C}$, hold for $5 \mathrm{~min}$. Cholesterol (Sigma) was used as internal standard.

\section{Monoterpenoid Extraction and Quantification}

The cells from a stationary phase culture in $150 \mathrm{~mL}$ minimum medium were harvested by centrifugation $(5,000 \mathrm{~g}$ for $5 \mathrm{~min})$. The culture supernatant was adjusted to $\mathrm{pH} 8.5$ with $\mathrm{KOH}$ (Merck) and $40 \mathrm{mg}$ eugenol (>99\%, Fluka) was added to evaluate extraction efficiency. The mixture $(40 \mathrm{~mL})$ was purified through a Bond Elut C18 $(5 \mathrm{~g})$ SPE cartridge (Varian) conditioned with methanol $(3 \times 10 \mathrm{~mL})$ and water $(3 \times 10 \mathrm{~mL})$. After washing with water $(10 \mathrm{~mL})$, terpenols were eluted with $10 \mathrm{~mL}$ methylene chloride (Fluka). The sample was dehydrated with anhydrous $\mathrm{Na}_{2} \mathrm{SO}_{4}$ (Merck), concentrated to about $500 \mu \mathrm{l}$ under a stream of nitrogen, $20 \mu \mathrm{g} m$-cresol (>99.9\%, Fluka) was added as internal standard and the sample was stored at $-20^{\circ} \mathrm{C}$ prior to GC-MS analysis. Extraction of intracellular monoterpenoids was performed on the yeast pellet obtained after centrifugation. Cells were crushed with glass beads in $1 \mathrm{~mL}$ of water and after addition of eugenol $(40 \mu \mathrm{g})$, the mixture was extracted three times with $n$-pentane $(>99 \%$, Merck). Terpenol extracts were analyzed by capillary GCMS using a Varian 3300 chromatograph equipped with oncolumn injector coupled to an HP 5970 mass-selective detector in electron ionization mode at $70 \mathrm{eV}$. Mass spectra were recorded by means of HP ChemStation version A.03.00 and compared with those of reference compounds also used for quantification. Separation of $1 \mu \mathrm{L}$ of extract was carried out on a $60-\mathrm{m}, 0.32-\mathrm{mm}$ ID, $0.5-\mathrm{mm}$ DB-Wax (J\&W Scientific) capillary column using helium as carrier gas at a constant pressure of 9 psi, 620 mbar. Separation conditions were: initial injector temperature of $70^{\circ} \mathrm{C}$ for $30 \mathrm{~s}$, then increased to $220^{\circ} \mathrm{C}$ at $160{ }^{\circ} \mathrm{C} / \mathrm{min}$; oven temperature was programmed without initial hold time at a rate of $2.7^{\circ} \mathrm{C} /$ min, from 67 to $235^{\circ} \mathrm{C}$ (hold for $5 \mathrm{~min}$ ). Total amounts of linalool, citronellol, and geraniol were determined using linear calibration curves with an $R^{2}$ value 0.98 over the concentration range from 0 to $200 \mathrm{mg} / \mathrm{mL}$.

\section{Geraniol Stability}

Geraniol $(100 \mu \mathrm{g} / \mathrm{L})(98 \%$, Sigma) was added to $100 \mathrm{~mL}$ citrate/ $\mathrm{HCl}$ buffer $(10 \mathrm{mM} \mathrm{pH} \mathrm{3.4)}$ or phosphate buffer (10 mM pH 7) in $125 \mathrm{~mL}$ bottles. Bottles were hermetically closed and left at room temperature for 65 days. Samples of $2 \mathrm{~mL}$ were taken at day 0-3-8-20-65 and eugenol $(100 \mu \mathrm{g})$ was added to evaluate extraction efficiency with $2 \mathrm{~mL}$ dichloromethane. The mixture was vortexed for $1 \mathrm{~min}$. The organic phase was dehydrated with anhydrous $\mathrm{Na}_{2} \mathrm{SO}_{4}$, and a $1.5 \mathrm{~mL}$ sample was stored at $-20{ }^{\circ} \mathrm{C}$ prior to GC-MS analysis. GC-MS analysis was performed as previously described.

\section{FPPS Model Building}

Homology modeling was done using Modeller (Sali and Blundell, 1993) with 1FPS, avian farnesyl diphosphate synthase, as template (Tarshis et al., 1994). 1FPS was selected because it ranked first when our yeast sequence was blasted against the sequences in the PDB (46\% identities, 66\% similarities and 2 short gaps over 348 residues). 1UBV, IUBW, 1UBX, and 1UBY which correspond to avian FPPSs mutated in position 112 and 113 (F112A/F113S) ranked very close to the wild type sequence (Tarshis et al., 1996). These structures were used as well because they show, respectively, the enzymes with empty active site pocket, with bound GPP and $\mathrm{Mg}^{++}$, with bound FPP and $\mathrm{Mg}^{++}$, and with bound DMAPP and $\mathrm{Mg}^{++}$. Yeast FPPS sequence was aligned with Avian FPPS using ClustalX (Thompson et al., 1997). The visual analysis of the model and the illustrations were done with PyMOL (DeLano, 2002).

\section{Results}

\section{Assessment of K197 Mutations on Cell Growth}

Yeast K197M and K197Q mutated strains were not obtained despite several attempts. It is noteworthy that K197L mutation was not straightforward as several attempts were necessary to obtain the mutated strain. We used yeast FPPS modeling to evaluate the putative structural effect of K197 mutations on the catalytic site (Fig. 1).

Introduction of a K197 mutation in FPPS resulted for 16 strains in an increase in recorded doubling time compared to the original strain (K197K) doubling time, and only one strain $(\mathrm{K} 197 \mathrm{H})$ had no observed statistically significant change (Table I). No strain had a statistically significant faster doubling time than the original K197K strain. The most impaired K197 replacements correspond to amino acids with long aliphatic chains. Increasing the chain length K197G-K197A-K197V-K197L resulted in an increase in doubling times, which was also observed for GES-expressing strains. Surprisingly, K197I does not exhibit the same pattern as K197L and leads to doubling times similar to K197G. Several mutants tested show little effect on doubling times although the amino acids incorporated have very different physicochemical properties. No simple correlation could be found between doubling time and a single property (mass, charge or hydrophobicity). Rather similar doubling times were observed for very different amino acids. 
Table I. Doubling time (h) and sterol content (\% dry weight) of FPPS mutated yeast strains untransformed (left part) or transformed (right part) with a GES plasmid.

\begin{tabular}{|c|c|c|c|c|c|}
\hline Strain & Doubling Time $(\mathrm{h})$ & Sterol content & Strain & Doubling Time $(\mathrm{h})$ & Sterol content \\
\hline K & $4.14 \pm 0.07$ & $0.37 \pm 0.04$ & AE K & $5.62 \pm 0.86$ & $0.33 \pm 0.06$ \\
\hline $\mathrm{H}$ & $4.92 \pm 0.94$ & $0.41 \pm 0.08$ & $\mathrm{AE} \mathrm{H}$ & $5.08 \pm 0.56$ & $0.37 \pm 0.11$ \\
\hline $\mathrm{P}$ & $4.99 \pm 0.76$ & $0.43 \pm 0.09$ & $\mathrm{AE} P$ & $5.28 \pm 0.47$ & $0.39 \pm 0.01$ \\
\hline $\mathrm{E}$ & $5.02 \pm 0.04$ & $0.59 \pm 0.06$ & $\mathrm{AE} \mathrm{E}$ & $5.75 \pm 0.26$ & $0.44 \pm 0.06$ \\
\hline $\mathrm{D}$ & $5.06 \pm 0.18$ & $0.52 \pm 0.07$ & $\mathrm{AE} \mathrm{D}$ & $6.64 \pm 0.86$ & $0.34 \pm 0.10$ \\
\hline $\mathrm{F}$ & $5.78 \pm 0.37$ & $0.58 \pm 0.06$ & $\mathrm{AE} \mathrm{F}$ & $10.63 \pm 2.74$ & $0.53 \pm 0.09$ \\
\hline W & $5.95 \pm 0.45$ & $0.56 \pm 0.02$ & $\mathrm{AE} \mathrm{W}$ & $9.48 \pm 3.81$ & $0.52 \pm 0.01$ \\
\hline Y & $5.97 \pm 0.58$ & $0.50 \pm 0.12$ & $\mathrm{AE} \mathrm{Y}$ & $10.07 \pm 3.47$ & $0.58 \pm 0.02$ \\
\hline $\mathrm{N}$ & $6.22 \pm 0.57$ & $0.49 \pm 0.04$ & $\mathrm{AE} \mathrm{N}$ & $7.54 \pm 0.85$ & $0.47 \pm 0.05$ \\
\hline $\mathrm{R}$ & $6.25 \pm 0.95$ & $0.37 \pm 0.07$ & AE R & $6.61 \pm 1.57$ & $0.37 \pm 0.04$ \\
\hline I & $6.35 \pm 0.56$ & $0.51 \pm 0.1$ & $\mathrm{AE} \mathrm{I}$ & $6.42 \pm 0.57$ & $0.50 \pm 0.03$ \\
\hline $\mathrm{C}$ & $6.76 \pm 0.75$ & $0.55 \pm 0.02$ & $\mathrm{AE} \mathrm{C}$ & $8.38 \pm 1.39$ & $0.43 \pm 0.07$ \\
\hline S & $7.00 \pm 0.80$ & $0.40 \pm 0.04$ & $\mathrm{AE} \mathrm{S}$ & $7.55 \pm 0.93$ & $0.40 \pm 0.08$ \\
\hline G & $7.53 \pm 0.67$ & $0.52 \pm 0.05$ & $\mathrm{AE} \mathrm{G}$ & $6.42 \pm 0.41$ & $0.45 \pm 0.02$ \\
\hline $\mathrm{T}$ & $7.74 \pm 0.70$ & $0.49 \pm 0.06$ & $\mathrm{AE} \mathrm{T}$ & $7.13 \pm 0.79$ & $0.52 \pm 0.01$ \\
\hline $\mathrm{V}$ & $9.52 \pm 2.00$ & $0.55 \pm 0.04$ & $\mathrm{AE} \mathrm{V}$ & $11.89 \pm 4.18$ & $0.63 \pm 0.02$ \\
\hline A & $9.75 \pm 1.39$ & $0.54 \pm 0.07$ & $\mathrm{AE} \mathrm{A}$ & $9.12 \pm 1.26$ & $0.49 \pm 0.06$ \\
\hline $\mathrm{L}$ & $17.61 \pm 3.85$ & $0.56 \pm 0.05$ & $\mathrm{AE} \mathrm{L}$ & $21.06 \pm 2.81$ & $0.70 \pm 0.08$ \\
\hline
\end{tabular}

Doubling time results are the mean of 3-5 experiments \pm standard deviation and sterol content are the mean of 10 experiments \pm standard deviation.

The effect of GES expression on doubling time was assessed in minimal medium (Table I). It resulted for six strains in an increase in doubling time compared to the strains untransformed with GES (K197F, Y, W, K, D, E). No statistically significant changes were observed for 11 strains (K197A, V, L, I, P, C, S, T, R, H, N). One strain had a statistically significant faster doubling time after GES expression (K197G).

Yeast cells exhibited a great variety of sterol content (Table I). The lowest sterol content was observed for strain K197K with $0.37 \%$ dry weight content, whereas the highest sterol content was observed for strain K197E with $0.59 \%$. Introduction of a K197 mutation resulted for 12 strains in an increase in sterol content compared with the original K197K sequence (K197G, A, V, L, I, Y, W, C, T, D, E, N) and no statistically significant changes were observed for only five strains (K197P, F, S, R, H). No strain had statistically significant lower sterol content than the original K197K strain. Basic amino acids $\mathrm{K}, \mathrm{R}, \mathrm{H}$ give the lowest sterol content. High sterol contents were observed with aliphatic G, A, V, L, I,; aromatic F, Y, W; sulfur C, and acidic D, E amino acid families. A mixed response was observed with aliphatic hydroxyl amino acids (S low, T high) and average levels with the unusual aliphatic $\mathrm{P}$ and the amide N. Sterol contents observed with or without GES expression were relatively close. Expression of GES resulted for two strains $(\mathrm{K} 197 \mathrm{~V}, \mathrm{~L})$ in an increase in recorded sterol content compared to the strains untransformed with GES and no statistically significant changes were observed for 11 strains (K197G, A, I, P, F, Y, S, T, K, R, H, N). Four strains had statistically significant lower sterol content after GES expression (K197W, C, D, E). Some strains somehow exhibited a significant decrease of sterol content (K197G, C,
D, E) and shifted from a high sterol content to a low or average sterol content.

It is noteworthy that yeast cells with impaired doubling times of more than $9.25 \mathrm{~h}$ also had statistically significant higher sterol content than the original K197K strain (Fig. 2). These strains correspond to aliphatic K197A, V, L strains either untransformed or transformed to express GES and to aromatic K197F, Y, W strains transformed to express GES. Strains with doubling times between 4.9 and 9.25 h exhibited a great variety of sterol content.

\section{Effects of K197 Mutations and of GES Expression on Monoterpenols Content}

Yeast cells exhibited a great variety of monoterpenols production in YNB medium: geraniol, linalool and citronellol were the main products whereas a small production of nerol and $\alpha$-terpineol was detected for some strains $(2-20 \mu \mathrm{g} / \mathrm{mL}$, data not shown) (Table II). Introduction of a $\mathrm{K} 197$ mutation resulted for 10 strains (K197G, A, L, C, S, T, R, D, E, N) in an increase in monoterpenol content compared to the original K197K sequence, no statistically significant increase was observed for seven strains (K197V, I, P, F, Y, W, H). Geraniol and linalool were produced in similar quantities, whereas almost no production of citronellol was detected.

Table II also shows that expression of GES could greatly enhance (10-20 fold) monoterpenol production and resulted in a more contrasted profile variation between yeasts with high monoterpenol content (K197G, A, L, C, S, $\mathrm{T}, \mathrm{D}, \mathrm{E})$ and yeasts with almost no monoterpenol content (K197I, P, F, Y, W, K, H, N). Only two strains (K197V, R) 


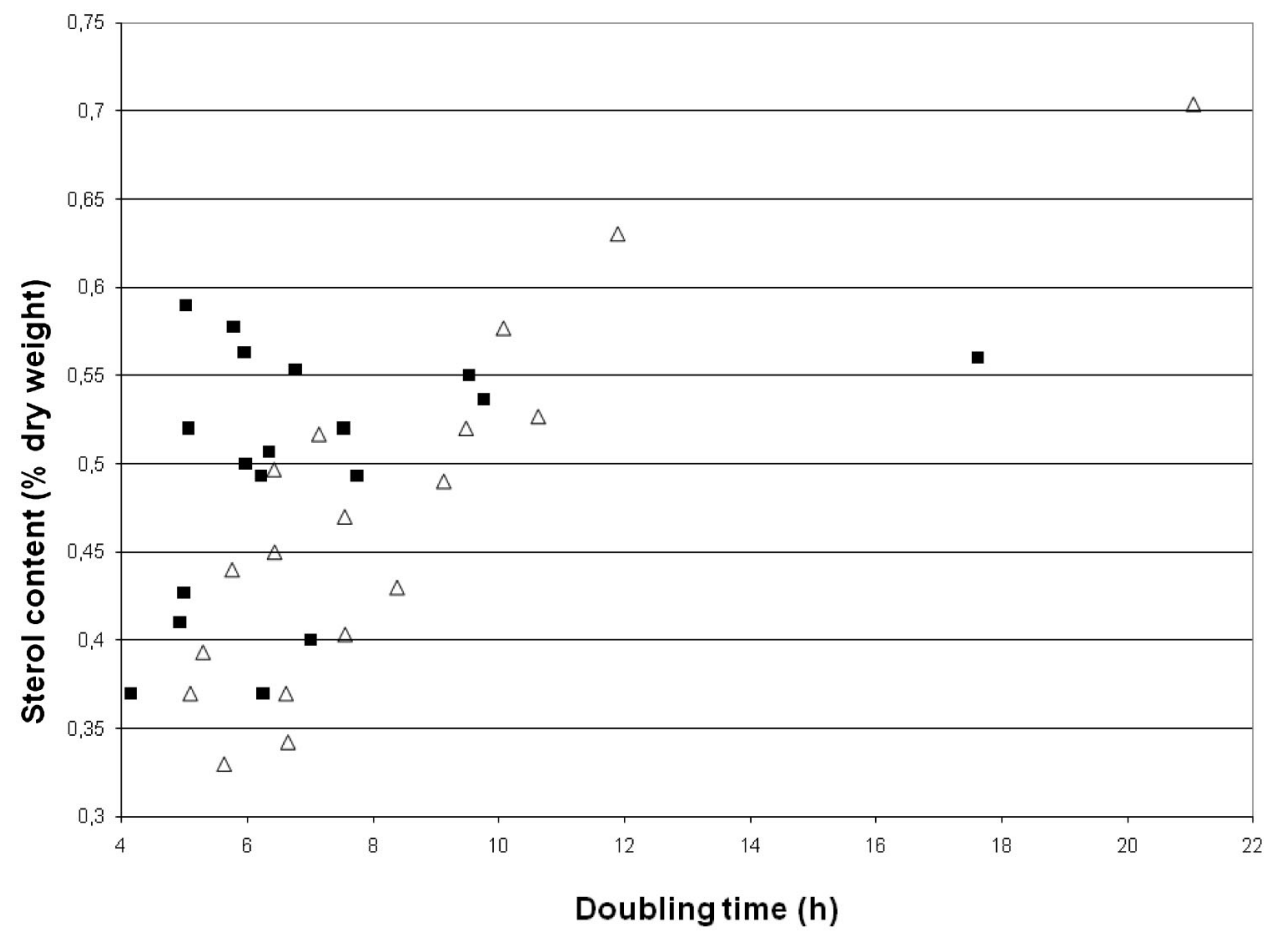

Figure 2. Relation between doubling time and sterol content of FPPS mutated yeast strains untransformed $(\boldsymbol{\square})$ or transformed $(\Delta)$ with a GES plasmid.

had intermediate monoterpenol content. A linear relationship was observed between the basic production and that upon GES expression for strains with high or intermediate monoterpenol content. Expression of GES had little or no effect on linalool profiles compared to the strains untransformed with GES but had a great impact on geraniol and citronellol content. Nerol and $\alpha$-terpineol were not produced in sufficient quantities to evaluate a possible modification of production. A more detailed analysis of the results also showed that for aliphatic amino acids, the decreasing monoterpenol order production is K197GK197A-K197L-K197V, with K197I standing out with no

Table II. Monoterpenol content ( $\mu \mathrm{g} / \mathrm{L})$ of FPPS mutated yeast strains untransformed (left part) or transformed (right part) with a GES plasmid.

\begin{tabular}{|c|c|c|c|c|c|c|c|c|c|}
\hline Strain & Geraniol & Linalool & Citronellol & Total monoterpenol & Strain & Geraniol & Linalool & Citronellol & Total monoterpenol \\
\hline G & $235 \pm 22$ & $232 \pm 8$ & $13 \pm 2$ & $480 \pm 28$ & $\mathrm{AE} \mathrm{G}$ & $5,366 \pm 3,425$ & $123 \pm 106$ & $68 \pm 48$ & $5,557 \pm 3,572$ \\
\hline S & $167 \pm 74$ & $201 \pm 56$ & $4 \pm 6$ & $372 \pm 130$ & $\mathrm{AE} \mathrm{S}$ & $5,090 \pm 2,483$ & $136 \pm 155$ & $292 \pm 211$ & $5,518 \pm 2,556$ \\
\hline A & $284 \pm 83$ & $360 \pm 117$ & $12 \pm 2$ & $656 \pm 199$ & $\mathrm{AE} \mathrm{A}$ & $4,593 \pm 1,507$ & $143 \pm 124$ & $112 \pm 60$ & $4,849 \pm 1,572$ \\
\hline $\mathrm{E}$ & $121 \pm 9$ & $78 \pm 57$ & $2 \pm 3$ & $201 \pm 61$ & $\mathrm{AE} \mathrm{E}$ & $3,725 \pm 370$ & $313 \pm 165$ & $152 \pm 84$ & $4,190 \pm 619$ \\
\hline $\mathrm{T}$ & $183 \pm 54$ & $181 \pm 17$ & $5 \pm 4$ & $369 \pm 71$ & $\mathrm{AE} \mathrm{T}$ & $3,517 \pm 1,076$ & $273 \pm 292$ & $160 \pm 75$ & $3,950 \pm 825$ \\
\hline $\mathrm{D}$ & $136 \pm 9$ & $133 \pm 19$ & $3 \pm 1$ & $272 \pm 28$ & AE D & $3,053 \pm 1,283$ & $172 \pm 114$ & $146 \pm 24$ & $3,371 \pm 1,291$ \\
\hline $\mathrm{C}$ & $120 \pm 11$ & $96 \pm 14$ & $8 \pm 3$ & $224 \pm 27$ & $\mathrm{AE} \mathrm{C}$ & $2,241 \pm 1,223$ & $216 \pm 225$ & $142 \pm 121$ & $2,599 \pm 1,568$ \\
\hline $\mathrm{L}$ & $118 \pm \pm 20$ & $130 \pm 6$ & $13 \pm 7$ & $262 \pm 14$ & $\mathrm{AE} \mathrm{L}$ & $1,840 \pm 361$ & $258 \pm 195$ & $99 \pm 18$ & $2,197 \pm 209$ \\
\hline $\mathrm{V}$ & $60 \pm 45$ & $45 \pm 35$ & ND & $105 \pm 73$ & $\mathrm{AE} \mathrm{V}$ & $875 \pm 488$ & $152 \pm 95$ & $46 \pm 21$ & $1,073 \pm 598$ \\
\hline $\mathrm{R}$ & $104 \pm 33$ & $91 \pm 7$ & $12 \pm 16$ & $207 \pm 24$ & AE R & $350 \pm 65$ & $72 \pm 55$ & $29 \pm 33$ & $450 \pm 55$ \\
\hline $\mathrm{F}$ & $28 \pm 8$ & $11 \pm 8$ & $1 \pm 1$ & $39 \pm 15$ & $\mathrm{AE} \mathrm{F}$ & $91 \pm 26$ & $13 \pm 7$ & $1 \pm 2$ & $105 \pm 20$ \\
\hline W & $33 \pm 32$ & $31 \pm 43$ & ND & $65 \pm 37$ & AE W & $51 \pm 56$ & $5 \pm 3$ & $10 \pm 12$ & $66 \pm 44$ \\
\hline $\mathrm{Y}$ & $5 \pm 7$ & ND & ND & $3 \pm 6$ & AE Y & $53 \pm 15$ & $7 \pm 5$ & ND & $60 \pm 19$ \\
\hline $\mathrm{K}$ & $10 \pm 3$ & $2 \pm 3$ & ND & $12 \pm 6$ & $\mathrm{AE} \mathrm{K}$ & $30 \pm 12$ & $21 \pm 16$ & ND & $49 \pm 17$ \\
\hline I & $12 \pm 3$ & $10 \pm 9$ & ND & $21 \pm 11$ & $\mathrm{AE} \mathrm{I}$ & $35 \pm 28$ & $1 \pm 1$ & ND & $48 \pm 17$ \\
\hline $\mathrm{N}$ & $65 \pm 9$ & $85 \pm 14$ & ND & $150 \pm 23$ & AE N & $43 \pm 23$ & $5 \pm 8$ & ND & $47 \pm 31$ \\
\hline $\mathrm{P}$ & ND & ND & $\mathrm{ND}$ & ND & AE P & $38 \pm 22$ & $9 \pm 9$ & ND & $47 \pm 26$ \\
\hline $\mathrm{H}$ & $5 \pm 5$ & $3 \pm 2$ & ND & $7 \pm 7$ & AE $\mathrm{H}$ & $41 \pm 23$ & $3 \pm 5$ & ND & $44 \pm 29$ \\
\hline
\end{tabular}

Terpenoids were extracted from minimal medium at stationary growth phase. Results are the mean of 3-5 experiments \pm standard deviation. ND: Not Detected. 
production. We were also able to detect a geraniol synthesis for the sulfhydryl C, the aliphatic hydroxyl ST, the acidic DE. On the other hand, we had no marked activity for the unusual aliphatic $\mathrm{P}$, the aromatic FYW, the basic KRH, and the amide $\mathrm{N}$. These results show clearly that the geraniol synthesis obtained through GES expression is linked to GPP availability.

To gain an appreciation for the potential chemical conversions of monoterpenols excreted in the YNB culture medium due to its acidic $\mathrm{pH}$, we monitored geraniol chemical conversion over a 65-day period (Table III). The results showed that geraniol to linalool conversion is $\mathrm{pH}$ dependent. At $\mathrm{pH} 3.4,20.6 \%$ geraniol was converted to linalool whereas at $\mathrm{pH} 7$ no conversion was observed. However, on a short period, until about 1 week, only a small (5\%) conversion of geraniol to linalool was observed, confirming that linalool arises from GPP and not from geraniol. A pH effect was also observed on geraniol conversion to nerol. At $\mathrm{pH} 3.4$, only $1.7 \%$ geraniol was converted to nerol whereas at $\mathrm{pH} 7.014 .6 \%$ conversion was observed. No conversion of geraniol to citronellol was observed at $\mathrm{pH} 3.4$ or $\mathrm{pH} 7.0$, indicating that citronellol arises in yeast from GPP or geraniol by an enzymatic conversion and not from geraniol via chemical conversion. A $\mathrm{pH}$ dependent degradation effect was also observed on total terpenol content as at pH $3.451 .9 \%$ terpenols remained whereas at $\mathrm{pH} 7.0$ only $22 \%$ terpenols remained. Geraniol final content was therefore $\mathrm{pH}$ dependent: at $\mathrm{pH}$ $3.4,27.5 \%$ geraniol remained whereas at $\mathrm{pH} 7.0$ only $5.9 \%$ geraniol remained. We observed a minor conversion of geraniol to citronellol and $\alpha$-terpineol.

\section{Discussion}

In this study we generated a set of FPPS mutants at position K197 and we wanted to address four points: (1) the growth properties of the mutants compared with the parent strain, (2) how this relates to sterol metabolism, (3) any structural information on FPPS enzymatic properties that could be inferred, and (4) the potential of these strains in relation to monoterpenol production.

Concerning the first point, FPPS gene is essential for S. cerevisiae (Anderson et al., 1989) and one could expect a lethal effect of K197 mutations affecting the catalytic site at an absolutely fixed position in a drastic way, and hence leading to a level of FPPS that could not sustain cellular metabolism. Surprisingly, our results show that only K197M and K197Q mutations either leading to a complete loss of activity, or affecting strongly FPP synthesis could not be obtained and may lead to lethality. They demonstrate that any of the other amino acids is tolerated at that position, although L may be somewhat problematic. We assessed the general fitness of these strains by monitoring the doubling time and comparing the results with that obtained for the K197 parental strain. Our results show that the best FPPS version is obtained as expected with the native version amino acid K197 (4.14 h). It has been previously demonstrated that about $40 \%$ of wt FPPS activity is sufficient to support normal growth of the mutant (Karst et al., 2004). We show here that any modification at this position has a strong effect on yeast doubling time except in $\mathrm{K} 197 \mathrm{H}$, the second fastest doubling strain. This is noteworthy as the 200 other FPPS sequences we tested in a multialignment harbor a $\mathrm{K}$ at the equivalent position. This would suggest that an increase of $20 \%$ in doubling time is largely sufficient for negative selection to operate.

A broad effect of the K197 mutations could be expected on cellular metabolism. There is increasing evidence that the amount of available farnesyl diphosphate substrate plays a key role in several yeast metabolites as intermediates of synthesis (i.e., ergosterol, heme A, ubiquinones, dolichols, and posttranslational farnesyl- and geranylgeranylation of proteins (Grabinska et al., 2007)). Considering the multiple roles played by these isoprenoids it is clear that the level of their synthesis must be strictly controlled to ensure optimal cell growth. The relation of FPPS mutations and these metabolites has however not been extensively studied in yeast, except in the case of ergosterol and dolichol, an isoprenoid lipid known mainly for its function in protein glycosylation. The Saccharomyces cerevisiae LB332 yeast strain bearing a K197E mutation in the ERG20 gene encoding farnesyl diphosphate synthase (FPPS) synthesizes significantly longer dolichols than the wild type strain FL100 (14-31 and 14-19 isoprene units, respectively). LB332 strain bearing a ERG20 K197E mutation have an increase in the size of the FPPS substrate binding pocket which decreases concentration of FPP over IPP. The activity

Table III. Stability of geraniol in acidic and neutral solutions over a 65-day period.

\begin{tabular}{|c|c|c|c|c|c|c|c|c|c|c|c|c|}
\hline \multirow[b]{2}{*}{ Day } & \multicolumn{6}{|c|}{$\mathrm{pH} 3.4$} & \multicolumn{6}{|c|}{$\mathrm{pH} 7.0$} \\
\hline & Geraniol & Linalool & Citronellol & Nerol & $\alpha$-Terpineol & $\begin{array}{c}\text { Total } \\
\text { Terpenols }\end{array}$ & Geraniol & Linalool & Citronellol & Nerol & $\alpha$-Terpineol & $\begin{array}{c}\text { Total } \\
\text { Terpenols }\end{array}$ \\
\hline 0 & 79.6 & ND & ND & ND & ND & 79.6 & 77.9 & ND & ND & ND & ND & 77.9 \\
\hline 3 & 75.9 & 2.5 & ND & ND & ND & 78.4 & 78.7 & ND & ND & ND & ND & 78.7 \\
\hline 8 & 70.9 & 6.3 & ND & 1 & ND & 78.2 & 62.2 & ND & ND & 2.9 & ND & 65.1 \\
\hline 20 & 55.4 & 11.9 & ND & 1.2 & ND & 68.6 & 40.9 & $\mathrm{ND}$ & ND & 10 & ND & 50.9 \\
\hline 65 & 27.5 & 20.6 & ND & 1.7 & 2 & 51.9 & 5.9 & $\mathrm{ND}$ & 1.5 & 14.6 & ND & 22 \\
\hline
\end{tabular}

Results are given as $\mathrm{mg} / \mathrm{L}$. ND, Not Detected. 
of cis-prenyltransferase involved in dolichol synthesis is shifted toward longer products when the IPP/FPP ratio is increased (Plochocka et al., 2000).

In the present study we decided to concentrate on the effect on cellular sterol metabolism, the second point of this discussion. Ergosterol is an essential component of the yeast plasma membrane that affects fluidity, permeability, and the activity of some membrane-bound enzymes. It is also a major component of secretory vesicles (Bagnat et al., 2000), plays a role in endocytosis (Heese-Peck et al., 2002), and participates in mitochondrial respiration (Altmann and Westermann, 2005). Ergosterol is also involved in oxygen sensing. It has previously been shown that the amount of available FPP substrate for ergosterol synthesis is directly regulated by FPPS activity. Overexpression of the FPPS gene (Szkopinska et al., 2000) and other experiments using mutated FPPS with decreased activity (Blanchard and Karst, 1993; Karst et al., 2004) indicates that FPPS influences the level of ergosterol synthesized. Similar to cholesterol in mammalian systems, it is the main end-product of IPP and DMAPP produced by the MVA pathway. Therefore one could expect that a decrease or increase in FPPS activity should lead to the concomitant decrease or increase of ergosterol synthesis. Data previously obtained on in vitro activity of FPPS using a CC25 yeast strain, a leaky FPPS defective strain exhibiting an additional mevalonate kinase mutation, have shown that at $42 \%$ wt FPPS activity, the amount of cell ergosterol was similar to that of the weight strain (Karst et al., 2004). At 20\% wt FPPS activity (K197R), the amount of ergosterol was decreased to half of the weight strain and, interestingly it remains practically unchanged at that level down to 3\% wt FPPS activity (K197V). In another study, it has been shown that a 6-fold increase in FPPS activity results in an increase of $32 \%$ in sterol level (Szkopinska et al., 2000). Taken together these results mean that a minor change in ergosterol content could reflect a major change in FPPS activity. In the present study we found a variation of up to $50-60 \%$ in ergosterol levels compared with the original K197K strain, thus suggesting a major modification in FPPS activity.

It was also possible to find a correlation between slow growth rates and high sterol contents for some strains. This was observed for aliphatic K197A, V, L strains, either untransformed or transformed to express GES. We do not know if the high content of sterol in these strains is a result of their slow growth or a result of different FPPS activities. Rather complex mechanisms may be at work as suggested by the aromatic K197F, Y, W strains also exhibiting an unexplained shift upon expression of GES, as their doubling time increased despite having no monoterpenol synthesis. It is possible to speculate that these K197F, Y, W strains release a very low amount of cytoplasmic GPP and that this GPP is depleted upon expression of the GES. This would suggest a yet unassigned role for GPP in yeast metabolism.

Concerning the third point, molecular modeling of the yeast FPPS gives us some insight into the behavior of our mutants. The K197 position of the residue close to the surface, on the opposite side of the substrate relative to IPP, allows K197 replacement without affecting in a drastic way the organization of amino acids in surrounding positions. The situation on the surface allows residues of various sizes (from the tiny $\mathrm{G}$ to the bulky W), charges (from acidic $\mathrm{E}$ to basic R), and polarities to be tolerated. The fact that sterol content is lower with $\mathrm{K}, \mathrm{R}$, and $\mathrm{H}$ than with the other amino acids suggests that the presence of amino groups is important but not vital as shown by the viability of the yeast mutants. The $3 \mathrm{D}$ model shows that the substrate diphosphate moiety is held in position by K197 and K254 which hold one phosphate each and act like a clamp (Fig. 1). Substituting one or the other may release the corresponding phosphate, resulting in a floating (wobbling) substrate that remains active though much less reactive. The $3 \mathrm{D}$ model neither suggests why Q or M are not tolerated, nor why I and $\mathrm{L}$ behave so differently.

Concerning the fourth point, the production of monoterpenols by Saccharomyces cerevisiae had been uncertain until the characterization of a K197E FPPS yeast mutant excreting geraniol and linalool (Chambon et al., 1990; Chambon et al., 1991). In a previous study we used this mutant strain and showed that it permits to increase monoterpenol production after heterelogous expression of GES from Ocimum basilicum (Oswald et al., 2007). We did however not know if this K197E mutation was the best to generate a pool of available GPP for monoterpenol synthesis. The present study firstly showed that several K197 mutations could strongly improve monoterpenol production as several strains (K197G, A, S, T) exhibited a marked increase in production. Further expression of GES resulted in markedly increased levels of monoterpenols for K197G, A, V, L, S, C, T, R, D, E mutated FPPS strains that reached up to about $5 \mathrm{mg} / \mathrm{L}$ whereas K197I, P, F, Y, W, K, H, N showed no increase in monoterpenol content. It should be noted that monoterpenol production obtained with GES heterologous expression is directly linked to the production observed in absence of GES. That demonstrates clearly that the mutations in FPPS permit an increase in the available GPP substrate for GES.

The analysis of the monoterpenols released in culture medium of mutant strains shows mainly geraniol and linalool at about the same level and small amounts of citronellol (Table II). The expression of GES in these strains increased strongly and specifically geraniol level leading to a ratio of geraniol/linalool of about 20:1. This is in good agreement with the product specificity of the geraniol synthase of $O$. basilicum, as already described (Iijima et al., 2004). The formation of linalool and citronellol could be linked either to an endogenous yeast enzyme acting on GPP or geraniol, or to a chemical instability. It is noteworthy that allylic isoprenoid diphosphates are known to be unstable at acidic pH (Rittersdorf and Kramer, 1967) and that conversion of monoterpenols into each other can be acidcatalyzed (Cori et al., 1986). In yeast, vacuolar $\mathrm{pH}$ is $4-5$, generated by the vacuolar $\mathrm{H}^{+}$ATPase (Rachid et al., 2004). Comparison of monoterpenol profiles from untransformed 
mutated strains and transformed with GES mutated strains indicate that (1) conversion of GPP to geraniol is catalyzed by GES but is also a product deriving from the hydrolysis of GPP, (2) linalool derives mainly from GPP but not from geraniol. Indeed in our culture conditions for about 3 days, the conversion of geraniol to linalool will be of about only $3 \%$ suggesting that the conversion of GPP rather than that of geraniol is at the basis of the linalool observed and that no enzyme activity needs to be involved. Our results are slightly different from what had been described in another study indicating that $S$. cerevisiae could change through enzymatic reactions added geraniol to linalool in minimal medium (King and Dickinson, 2000) but are in concordance with another study of geraniol to linalool conversion in a wine must fermentation (Vaudano et al., 2004). (3) Citronellol can be obtained from GPP or geraniol by enzymatic conversion. It is known that conversion of geraniol to citronellol is stereospecific in S. cerevisiae (Gramatica et al., 1982); therefore, it is likely the product of an endogenous yeast oxido-reductase. Data from Table III indicate that in acidic conditions a small part of geraniol can be chemically converted to nerol and $\alpha$-terpineol but in our culture conditions nerol and $\alpha$-terpineol levels are too low to be statistically compared between strains.

In conclusion, we show here that six strains had both good monoterpenol production and doubling time increased by a factor less than 2 compared to the control strain (K197G, C, $\mathrm{S}, \mathrm{T}, \mathrm{D}, \mathrm{E})$. One can reasonably suppose that genomic integration of such kind of mutations will permit to obtain strains with good genetic stability and monoterpenols production capabilities. Our study therefore provides a choice of several strains for heterologous monoterpene synthase expressions, as well as genetic information about how to modify industrial yeast strains to optimize monoterpenols production.

\section{References}

Altmann K, Westermann B. 2005. Role of essential genes in mitochondrial morphogenesis in Saccharomyces cerevisiae. Mol Biol Cell 16:54105417.

Anderson MS, Yarger JG, Burck CL, Poulter CD. 1989. Farnesyl diphosphate synthetase. Molecular cloning, sequence, and expression of an essential gene from Saccharomyces cerevisiae. J Biol Chem 264:1917619184

Ashby MN, Edwards PA. 1990. Elucidation of the deficiency in two yeast coenzyme Q mutants. Characterization of the structural gene encoding hexaprenyl pyrophosphate synthetase. J Biol Chem 265:13157-13164.

Bagnat M, Keranen S, Shevchenko A, Simons K. 2000. Lipid rafts function in biosynthetic delivery of proteins to the cell surface in yeast. Proc Natl Acad Sci USA 97:3254-3259.

Becker DM, Guarante L. 1991. High efficiency transformation of yeast by electroporation. Methods Enzymol 194:182-187.

Berman HM, Westbrook J, Feng Z, Gilliland G, Bhat TN, Weissig H, Shindyalov IN, Bourne PE. 2000. The protein data bank. Nucleic Acid Res 28:235-242.

Bitsch F, Lefeuvre P, Bègue D, Bourgier E, Jahnke W, Rondeau JM. 2006. Understanding the binding mechanism of substrates and bisphosphonates with farnesyl pyrophosphate synthase-a microcalorimetry study. Bone 38:44-44.
Blanchard L, Karst F. 1993. Characterization of a lysine-to-glutamic acid mutation in a conservative sequence of farnesyl diphosphate synthase from Saccharomyces cerevisiae. Gene 125:185-189.

Carrau FM, Medina K, Boido E, Farina L, Caggero C, Dellacassa E, Versini G, Henschke PA. 2005. De novo synthesis of monoterpenes by Saccharomyces cerevisiae wine yeasts. FEMS Microbiol Lett 234:107115.

Chambon C, Ladeveze V, Oulmouden A, Servouse M, Karst F. 1990. Isolation and properties of yeast mutants affected in farnesyl diphosphate synthetase. Curr Genet 18:41-46.

Chambon C, Ladeveze V, Servouse M, Blanchard L, Javelot C, Vladescu B, Karst F. 1991. Sterol pathway in yeast. Identification and properties of mutant strains defective in mevalonate diphosphate decarboxylase and farnesyl diphosphate synthetase. Lipids 26:633-636.

Cori O, Chayet L, Perez LM, Bunton CA, Hachey D. 1986. Rearrangement of linalool, geraniol, nerol and their derivatives. J Org Chem 51:1310 1316.

DeLano WL. 2002. The PyMOL Molecular Graphics System (2002) on World Wide Web http://www.pymol.org

Grabinska KA, Palamarczyk G. 2002. Dolichol biosynthesis in the yeast Saccharomyces cerevisiae: an insight into the regulatory role of farnesyl diphosphate synthase. FEMS Yeast Res 2:259-265.

Grabinska KA, Magnelli P, Robbins PW. 2007. Prenylation of Saccharomyces cerevisiae Chs4p affects chitin synthase III activity and chitin chain length. Eukaryot Cell 6:328-336.

Gramatica P, Manitto P, Ranzi BM, Delbianco A, Francavilla M. 1982. Stereospecific reduction of geraniol to (R)-(+)-citronellol by Saccharomyces cerevisiae. Experimentia 38:775-776.

Heese-Peck A, Pilcher H, Zanolaci B, Wanatabe R, Daum G, Riezman H. 2002. Multiple functions of sterols in yeast endocytosis. Mol Biol Cell 13:2664-2680.

Hosfield DJ, Zhang Y, Dougan DR, Broun A, Tari LW, Swanson RV, Finn J. 2004. Structural basis for bisphosphonate-mediated inhibition of isoprenoid biosynthesis. J Biol Chem 279:8526-8529.

Karst F, Plochocka D, Meyer S, Szkopinska A. 2004. Farnesyl diphosphate synthase activity affects ergosterol level and proliferation of yeast Saccharomyces cerevisae. Cell Biol Int 28:193-197.

King A, Dickinson R. 2000. Yeast Biotransformation of monoterpene alcohols by Saccharomyces cerevisiae, Torulaspora delbrueckii and Kluyveromyces lactis. Yeast 16:499-506.

Kirby J, Keasling JD. 2009. Biosynthesis of plant isoprenoids: perspectives for microbial engineering. Annu Rev Plant Biol 60:335-355.

Koyama T, Obata S, Osabe M, Takeshita A, Yokoyama K, Uchida M, Nishino T, Ogura K. 1993. Thermostable farnesyl diphosphate synthase of Bacillus stearothermophilus: molecular cloning, sequence determination, overproduction, and purification. J Biochem (Tokyo) 113:355363.

Iijima Y, Gang DR, Fridman E, Lewinsohn E, Pichersky E. 2004. Characterization of geraniol synthase from the pelate glands of sweet basil. Plant Physiol 134:370-379.

Marcireau C, Guilloton M, Karst F. 1990. In vivo effects of fenpropimorph on the yeast Saccharomyces cerevisiae and determination of the molecular basis of the antifungal property. Antimicrob Agents Chemother 34:898-993.

Martin VJ, Pitera DJ, Withers ST, Newman JD, Keasling JD. 2003. Engineering of a mevalonate pathway in Escherichia coli for production of terpenoids. Nat Biotechnol 21:796-802.

Oswald M, Fischer M, Dirninger N, Karst F. 2007. Monoterpenoid biosynthesis in Saccharomyces cerevisiae. FEMS Yeast Res 7:413-421.

Plewniak F, Bianchetti L, Brelivet Y, Carles A, Chalmel F, Lecompte O, Mochel T, Moulinier L, Muller A, Muller J, Prigent V, Ripp R, Thierry JC, Thompson JD, Wicker N, Poch O. 2003. PipeAlign: a new toolkit for protein family analysis. Nucleic Acid Res 31:38293832.

Plochocka D, Karst F, Swiezewska E, Szkopinska A. 2000. The role of ERG20 gene (encoding yeast farnesyl diphosphate synthase) mutation in long dolichol formation. Molecular modeling of FPP synthase. Biochimie 82:733-738. 
Rachid A, Brett CL, Mukherjee S, Rajini R. 2004. Inhibition of sodium/ proton exchange by a Rab-GTPase-activating protein regulates endosomal traffic in yeast. J Biol Chem 279:4498-4506.

Reiling KK, Yoshikuni Y, Martin VJJ, Newman J, Bohlmann J, Keasling JD. 2004. Mono and diterpene production in Escherichia coli. Biotechnol Bioeng 87:200-212.

Rittersdorf W, Kramer F. 1967. Die hydrolyse von 2,3 dihydroterpenylphosphaten und pyrophosphaten. Tetrahedron 23:3023-3088.

Sali A, Blundell TL. 1993. Comparative protein modelling by satisfaction of spatial restraints. J Mol Biol 234:779-815.

Sanchez VM, Crespo A, Gutkind JS, Turjanski AG. 2006. Investigation of the catalytic mechanism of farnesyl pyrophosphate synthase by computer simulation. J Phys Chem B 110:18052-18057.

Servouse M, Karst F. 1986. Regulation of early enzymes of ergosterol biosynthesis in Saccharomyces cerevisiae. Biochem J 240:541-547.

Shiba Y, Paradise EM, Kirby J, Ro DK, Keasling JD. 2007. Engineering of the pyruvate dehydrogenase bypass in Saccharomyces cerevisiae for highlevel production of isoprenoids. Metab Eng 9:160-168.

Stanley Fernandez SM, Kellog BA, Poulter CD. 2000. Farnesyl diphosphate synthase. Altering the catalytic site to select for geranyl diphosphate activity. Biochemistry 39:15316-15321.

Szkopinska A, Plochocka D. 2005. Farnesyl diphosphate synthase; regulation of product specificity. Acta Biochim Pol 52:45-55.

Szkopinska A, Swiezewska E, Karst F. 2000. The regulation of activity of main mevalonic acid pathway enzymes: Farnesyl diphosphate synthase, 3-hydroxy-3-methylglutaryl-CoA reductase, and squalene synthase in yeast Saccharomyces cerevisiae. Biochem Biophys Res Commun 267: 473-477.
Takahashi S, Yeo Y, Greenhagen BT, McMullin T, Song L, MaurinaBrunker J, Rosson R, Noel JP, Chappell J. 2007. Metabolic engineering of sesquiterpene metabolism in yeast. Biotechnol Bioeng 97:170181.

Tarshis LC, Yan M, Poulter CD, Sacchettini JC. 1994. Crystal structure of recombinant farnesyl diphosphate synthase at 2.6-A resolution. Biochemistry 33:10871-10877.

Tarshis LC, Proteau PJ, Kellogg BA, Sacchettini JC, Poulter CD. 1996. Regulation of product chain length by isoprenyl diphosphate synthases. Proc Natl Acad Sci USA 93:15018-15023.

Thompson JD, Gibson TJ, Plewniak F, Jeanmougin F, Higgins DG. 1997. The CLUSTAL_X windows interface: flexible strategies for multiple sequence alignment aided by quality analysis tools. Nucleic Acid Res 25:4876-4882.

Vaudano E, Moruno GM, Di Stephano R. 2004. Modulation of geraniol metabolism during alcohol fermentation. J Inst Brew 110:213-219.

Vandermoten S, Haubruge E, Cusson M. 2009. New insights into shortchain prenyltransferases: structural features, evolutionary history and potential for selective inhibition. Cell Mol Life Sci 66:3685-3695.

Withers ST, Keasling JD. 2007. Biosynthesis and engineering of isoprenoid small molecules. Appl Microbiol Biotechnol 73:980-990.

Ye YF, Fujii M, Hirata A, Kawamukai M, Shimoda C, Nakamura T. 2007. Geranylgeranyl diphosphate synthase in fission yeast is a heteromer of farnesyl diphosphate synthase (FPS), fps1, and an FPS-like protein, spo9, essential for sporulation. Mol Biol Cell 18:3568-3581.

Zea L, Moreno J, Ortega JM, Medina M. 1995. Content of free terpenic compounds in cells and musts during vinification with three Saccharomyces cerevisiae races. J Agr Food Chem 43:1110-1114. 